\title{
Accuracy of casts produced from conventional and digital workflows: A qualitative and quantitative analyses
}

\author{
Jaafar Abduo* \\ Restorative Section, Melbourne Dental School, Melbourne University, Victoria, Australia
}

\begin{abstract}
PURPOSE. Comparing the accuracy of casts produced from digital workflow to that of casts produced from conventional techniques. MATERIALS AND METHODS. Whole arch alginate (ALG) and polyvinyl siloxane (PVS) impressions were taken with stock trays and custom trays, respectively. The ALG impressions were poured with type III dental stone, while the PVS impressions were poured with type IV dental stone. For the digital workflow, IOS impressions were taken and physical casts were produced by 3D printing. In addition, 3D printed casts were produced from images obtained from a laboratory scanner (LS). For each technique, a total of 10 casts were produced. The accuracies of the whole arch and separated teeth were virtually quantified. RESULTS. Whole arch cast accuracy was more superior for PVS followed by LS, ALG, and IOS. The PVS and ALG groups were inferior in the areas more susceptible to impression material distortion, such as fossae and undercut regions. The LS casts appeared to have generalized errors of minor magnitude influencing primarily the posterior teeth. The IOS casts were considerably more affected at the posterior region. On the contrary, the IOS and LS casts were more superior for single tooth accuracy followed by PVS and ALG. CONCLUSION. For whole arch accuracy, casts produced from IOS were inferior to those produced from PVS and ALG. The inferior outcome of IOS appears to be related to the span of scanning. For single tooth accuracy, IOS showed superior accuracy compared to conventional impressions. [J Adv Prosthodont 2019;11:138-46]
\end{abstract}

KEYWORDS: 3D printing; Impression; Trueness; Precision; Virtual

\section{INTRODUCTION}

Dental impression is a routine procedure for diagnosis, treatment planning, and fabrication of prosthesis. To serve its purpose, the impression should be accurate, practical, predictable, and easy to implement. The conventional method of taking an impression involves using a stock or custom tray and a paste-like material such as alginate (ALG) or poly-

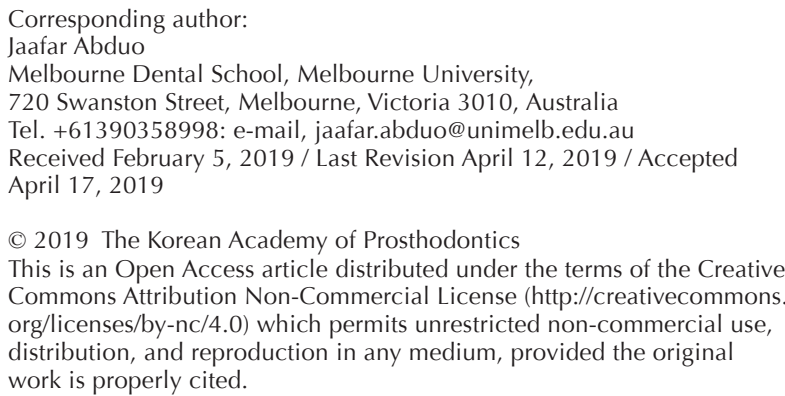

This study was supported by internal fund from Melbourne Dental School Research Higher. vinyl siloxane (PVS) ${ }^{1}$ However, the conventional impression methods are associated with limitations such as ongoing costs, patient discomfort, the requirement of well-fitting trays, and the necessity of pouring with dental stone. In addition, its quality depends on material handling, the distortion of the impression and stone material, and capturing all of the intraoral tissues. ${ }^{2-5}$

Recently, with the advancement of digital technologies, digital workflow to produce dental prostheses became a possibility. A key step of the digital workflow is the digital impressions with intraoral scanner (IOS). It is based on recording the arch details with an intraoral camera. A common application of digital workflow is the chairside computer-aided design and computer-aided manufacturing (CAD/CAM). This system became popular as it allows the production of the restoration at a single clinical appointment. Further, this workflow has the advantage of eliminating the need for impression and physical casts. However, its drawbacks are a restriction of the choice of materials and prosthesis span, and limited possibilities of veneering and customization of the restoration. ${ }^{6-10}$ 
To overcome these problems, more recently, a modified digital workflow was proposed, where the IOS is used to generate a virtual model of the arch that can be sent to manufacturing technicians or a milling centre where the restoration is produced. The advantages of this workflow are customization of the restoration by a skilled technician, and the possibility of implementing a more durable material as the restoration is produced by a milling centre and a commercial dental laboratory. ${ }^{11}$ Further, it can be used for longer span prostheses. In order to customize the restoration, a physical cast is required to ensure that accurate proximal and occlusal contacts are achieved. ${ }^{12-14}$ With the IOS-generated image, the file is converted to an stereolithography (STL) format and used to produce a physical cast by $3 \mathrm{D}$ printing. This cast is needed to relate the restoration to the adjacent teeth and opposing teeth, which allows the dental technician to customize the restoration. Thus, accuracy of the restorations produced by this workflow depends on IOS scanning, restoration design, and the printed cast accuracy. ${ }^{12,15-17}$

3D printing in dentistry gained popularity because of its versatility, material saving, and ability to produce workpieces of complex morphology, which make it ideal for large and detailed objects, such as casts, occlusal splints, and surgical guides. ${ }^{11}$ While earlier reports indicated that the complete workflow linked to the 3D printed cast is reliable for diagnostic purposes, it is still vulnerable to error that may influence the fit of the prosthesis. ${ }^{12,14-16,18,19}$ In addition, several studies indicated an inferior outcome of IOS in comparison to conventional impressions. ${ }^{7,8,10,17}$ Thus, it is important to critically evaluate the accuracy of $3 \mathrm{D}$ printed casts generated from IOS impressions in relation to their application (diagnostic and/or definitive prosthesis fabrication application), and isolate the factors that may influence the outcome of the accuracy of any digital workflow. Therefore, the aim of this study is to compare the accuracy (trueness and precision) of whole arch casts generated from IOS impressions and $3 \mathrm{D}$ printing against casts produced from ALG and PVS impressions. The casts produced from ALG impressions will represent casts routinely used for diagnostic purposes, while those generated from PVS impressions will resemble the ones used for definitive prostheses fabrication. In order to evaluate the impact of IOS, 3D printed casts from a laboratory scanner will be included. In addition, the comparison was executed at the level of separated teeth to determine the effect of span length. The null hypothesis is that all the workflows will produce casts of similar accuracy.

\section{MATERIALS AND METHODS}

A teaching Frasaco dentate maxillary model that has 16 teeth (Frasaco, Practicon, Greenville, NC, USA) was used for the study. A silicone mould (Dublisil 15, Dreve Dentamid, Unna, Germany) of the maxillary model was produced and used to fabricate polyurethane resin master model (Easycast, Bankstown, NSW, Australia). The resin model was scanned by a laboratory surface scanner (D-640 scanner, 3Shape Dental System, Copenhagen, Denmark) to generate a virtual master model that was used for all the subsequent evaluation steps. A total of 4 cast groups were evaluated in the study. The 2 groups of conventional casts were produced from alginate (ALG) impressions and polyvinyl siloxane (PVS) impressions. The other 2 groups were digital casts produced from 3D printing of STL images generated by an intraoral scanner (IOS) and a laboratory scanner (LS).

As a representation of diagnostic impressions, ALG impression material (Blueprint Xcreme, Dentsply, York, PA, USA) in a stock maxillary tray was used (Unident Gibling Dentate Tray, Durodent Dental, Bayswater, VIC, Australia). Prior to mixing the material, alginate tray adhesive (Fix Adhesive, Dentsply, York, PA, USA) was applied on the tray. The ALG impressions were poured up with type III dental stone (Unident Yellowstone, Unident, Werribee, Australia), which corresponds to the workflow routinely implemented for diagnostic casts.

The PVS group aimed to simulate the definitive impressions where custom trays and PVS impression materials are used. The custom trays were fabricated with light cure resin sheets (Light Curing Trayplates, Vertex, Haarlem, Netherlands). Two layers of base plate wax were applied on the master model to control the space between the tray and the model. The trays extended $3 \mathrm{~mm}$ beyond the gingival margin of the buccal and labial sulci. The definitive impressions were taken by a combination of light and medium body PVS impression materials (Extrude, Kerr, Lugano, Switzerland). PVS tray adhesive was applied on the custom tray (VPS Tray Adhesive, Kerr, Lugano, Switzerland). As per routine definitive impression procedure, the PVS impressions were poured with type IV dental stone (FujiRock, GC, Tokyo, Japan).

For all the impression materials, tray adhesives and stone materials, the manufacturers' instructions were followed. The impressions recorded all the teeth and the attached gingiva. The loaded trays were seated on the cast with even finger pressure to ensure complete seating of the tray on the master model. All the impressions were taken by a single operator. Each impression was repeated 10 times to produce 10 casts for each group.

For the recommended IOS digital flow, the master model was scanned 10 times by an intraoral scanner (Cerec Omnicam, Sirona, Bensheim, Germany) by the same operator who is experienced with the use of IOS systems. No scanning powder was applied prior to scanning. The scanning started by positioning the IOS camera on the most posterior molar on one side and ended on the most posterior molar on the other side. The camera moved through the arch in a zigzag motion to record the occlusal aspect followed by the palatal and buccal aspects. The generated virtual casts were converted to an STL format and transferred to a 3D printer (ProJet, 3510 DP Pro, 3D systems, Rock Hill, SC, USA) to produce 10 polyurethane casts. In order to evaluate the contribution of the printing step, the master model was scanned by a laboratory scanner 10 times and each generated STL image was imported to the same 3D printer to produce a total of 10 polyurethane casts (LS group). 
Every produced cast was scanned by the laboratory scanner (D-640 scanner) to generate virtual 3D casts. The accuracy was evaluated at 2 levels: arch level and tooth level. The arch accuracy was conducted to evaluate the suitability of recording the whole arch in terms of surface details and dimensions according to ISO standard. ${ }^{20}$ This involved trueness and precision calculation. The trueness is defined as deviation from the reference model, which was the initial image from the laboratory scanner, while precision is the deviation between different casts within the same group. Trueness provides information on error introduced from each technique, whereas precision quantifies the repeatability of each technique. The smaller the deviation value, the greater the accuracy. All the virtual casts were imported to a 3D rendering software (Geomagic Studio, 3D systems, Rock Hill, SC, USA). The virtual casts were trimmed to obtain the teeth and $2 \mathrm{~mm}$ height of attached gingivae. This would ensure that the comparison is restricted to the critical area that can be influenced by impression accuracy. Geomagic Studio software was then utilized to quantify the similarity of $3 \mathrm{D}$ models through image registration. This procedure consisted of point-to-point registration, global registration, and calculation of difference in distances between the surfaces of the models. The software oriented the models according to common anatomical landmarks, and then aligned the two virtual models according to the best-fit principles. Finally, it quantified the similarity between the master model and the virtual casts by averaging the absolute deviation of approximately 2000 random points on the surfaces to calculate the root mean square (RMS) value according to the following equation:

$$
R M S=\sqrt{\frac{\sum\left(R_{i}-C_{i}\right)^{2}}{n}}
$$

where $R_{i}$ is the spatial point of the reference model, $C_{i}$ is the same spatial point of the produced cast, and $n$ is the full number of points.

In addition, as a qualitative evaluation, colour-coded heat maps were extracted for each trueness measurement using the global registration function in the Geomagic Studio software. This step illustrated the location of the deviation between the virtual cast and the master model. Accurate fitting areas were represented in green. A positive discrepancy (warm colour) on the master model implies that the virtual cast is dimensionally larger than the master model, whereas a negative discrepancy (cool colour) implies the virtual casts are dimensionally smaller than the master model. In order to accurately compare the different colour maps,
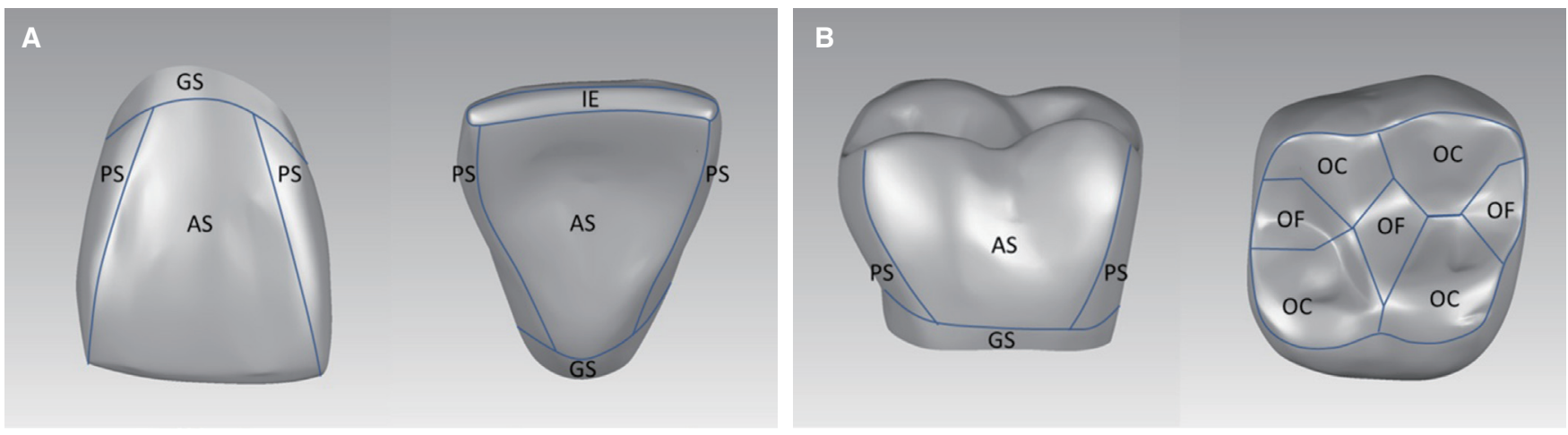

Fig. 1. Diagrams illustrating the segmentation of the different surfaces. (A) Anterior tooth, (B) Posterior tooth. AS =axial surface, $\mathrm{PS}=$ proximal surface, $\mathrm{GS}=$ gingival surface, $\mathrm{IE}=$ incisal edge, $\mathrm{OC}=$ occlusal cusp, and $\mathrm{OF}=$ occlusal fossa.

A

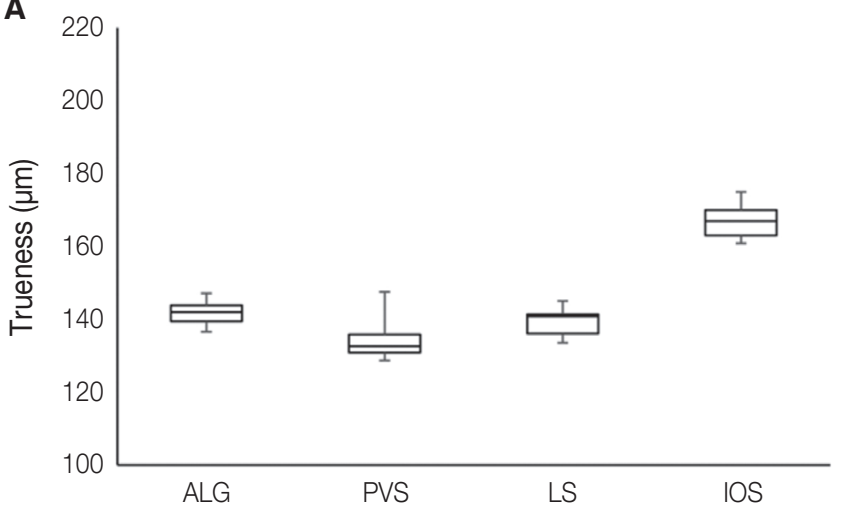

B

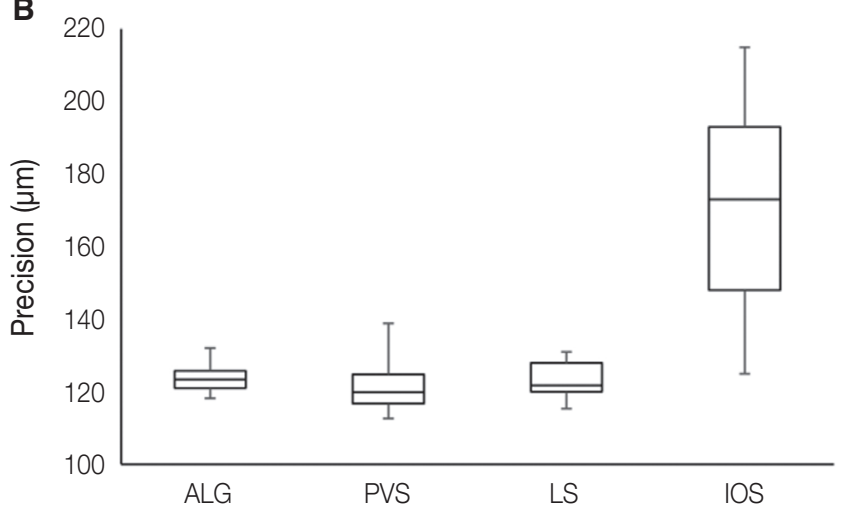

Fig. 2. Box plot diagrams comparing the accuracies of the different groups. (A) Trueness, (B) Precision. 
each coloured model was divided into 7 regions on the bases of the different surfaces on the teeth of the arch (Fig. 1): 6 incisal edges, 26 occlusal fossae, 32 occlusal cusps, 64 proximal surfaces, 32 axial surfaces, 30 interdental papillae, and 32 gingival surfaces. The frequency of deviation of the surfaces was counted and labelled according to the colour as follows: accurate (under $100 \mu \mathrm{m})$, minor deviation (100 $350 \mu \mathrm{m})$, moderate deviation $(350-600 \mu \mathrm{m})$, and major deviation (greater than $600 \mu \mathrm{m}$ ).

The tooth level evaluation aimed to quantify the accuracy of the short span extension of cast that corresponds to each individual tooth. The tooth accuracy was evaluated by computing the trueness. Each tooth of the master model was segmented and separated from the adjacent structures. Subsequently, each tooth was superimposed against its corresponding tooth from the cast image to measure the trueness. For the arch analysis, RMS values were calculated after each superimposition. The teeth were divided into 4 categories: (1) incisors, (2) canines, (3) premolars, and (4) molars.

The differences of mean RMS trueness and precision of the ALG, PVS, IOS, and LS were examined using one-way ANOVA, following confirmation of normality by ShapiroWilk test. A Tukey's post hoc test was performed whenever there was statistical difference. For the qualitative evaluation of each region of the arch, the different pattern of deviations was evaluated by the chi-square test. A two-way ANOVA was carried out for analyzing the trueness of different tooth groups. Two independent variables were included: (1) cast group (ALG, PVS, IOS and LS) and (2) tooth category (incisors, canine, premolars, and molars). For all the statistical tests, the level of significance was set to 0.05 .

\section{RESULTS}

In the relation to trueness, PVS was the most accurate $(134.7 \pm 6.2 \mu \mathrm{m})$, followed by LS $(139.1 \pm 3.9 \mu \mathrm{m})$, ALG $(141.7 \pm 3.3 \mu \mathrm{m})$, and IOS $(167.0 \pm 4.9 \mu \mathrm{m})$ respectively (Fig. 2A). There was no significant difference among the ALG, PVS, and LS. The IOS was significantly more inferior compared to the other groups $(P<.001)$. For the precision, generally similar values were observed for PVS $(121.9 \pm 7.0$ $\mu \mathrm{m})$, ALG $(123.6 \pm 3.4 \mu \mathrm{m})$, and LS $(123.3 \pm 4.3 \mu \mathrm{m})$ (Fig. 2B). The IOS $(170.9 \pm 27.9 \mu \mathrm{m})$ had a significantly inferior precision $(P<.001)$ than the other groups.

Fig. 3 illustrates examples of qualitative deviation pattern of the different surfaces. The deviation pattern appears to be influenced by the surface and the cast production technique. Overall, there was a general similarity between ALG and PVS. The conventional groups appeared to be more accurate on the occlusal and incisal aspects, but suffered from more errors at the axial surfaces, proximal surfaces, and gingival surfaces, which seemed to be related to the presence of undercuts. The LS had more systematic errors of a generally minor magnitude. The LS casts appeared slightly narrower at the posterior regions. The IOS severity of errors increased at the posterior teeth on the occlusal and axial surfaces, and the casts were generally wider than the master model.

For the occlusal fossae (Fig. 4A), all the groups showed a tendency for positive errors, in which the fossae tended to be shallower than the fossae of the master model. The LS appeared to be more accurate at the occlusal fossae, while the ALG was the most inferior. The IOS had a greater prev-
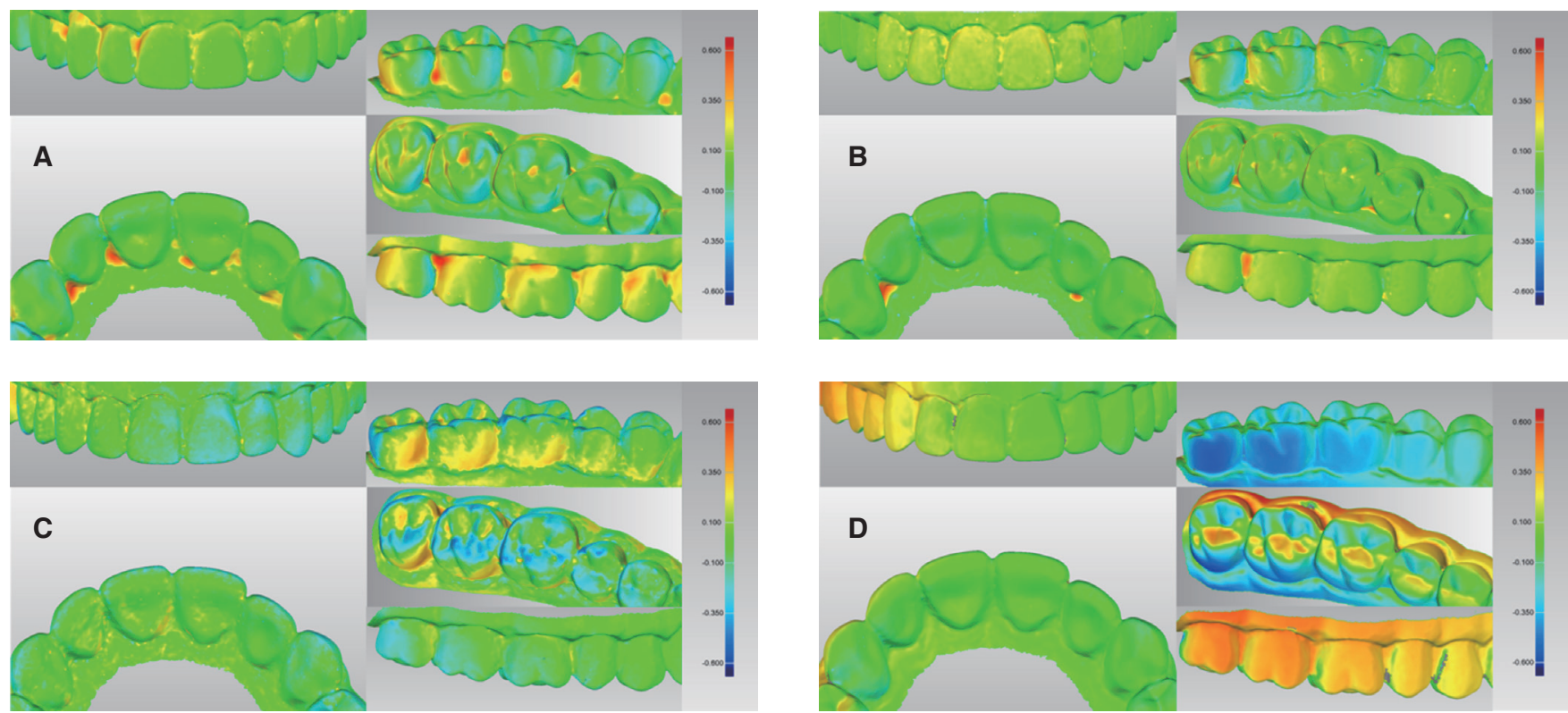

Fig. 3. Examples of heat maps of different surfaces for each group. (A) ALG, (B) PVS, (C) LS, (D) IOS. The ALG and PVS tend to show distortion at the interdental papillae, occlusal fossae, and gingival surfaces. The LS casts suffered from generalized error of minor magnitude and the casts appeared narrower than the original model. The IOS suffered from widening that primarily affected the accuracy of the posterior segments. 
A

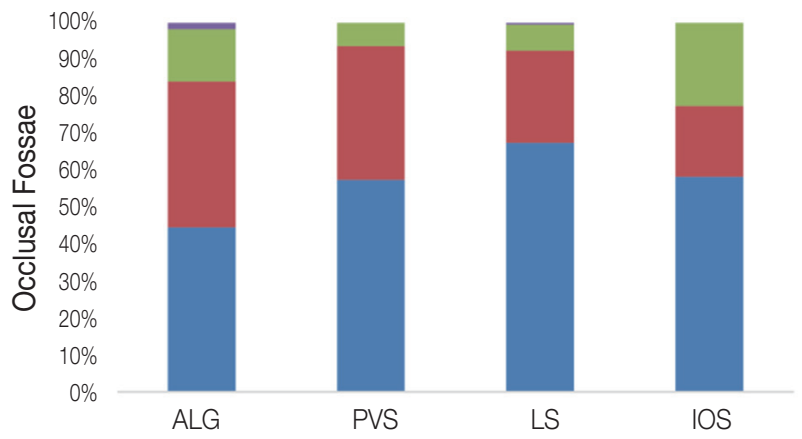

=Accurate $\quad$ Minor Deviation $=$ Moderate Deviation $\quad$ - Major Deviation

\section{C}

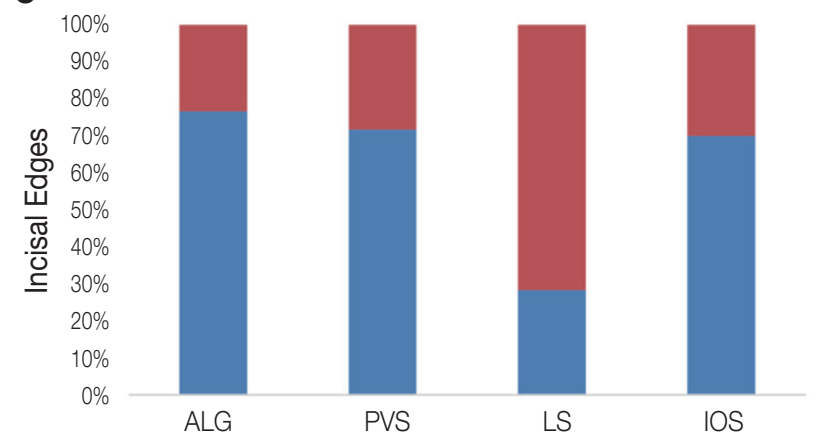

-Accurate $\quad$ Minor Deviation $\quad$ m Moderate Deviation $\quad$ Major Deviation

E

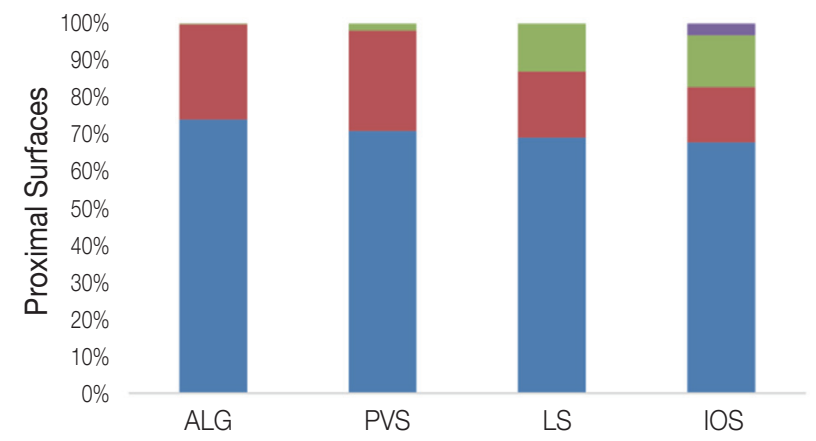

- Accurate $=$ Minor Deviation $=$ Moderate Deviation $=$ Major Deviation

G

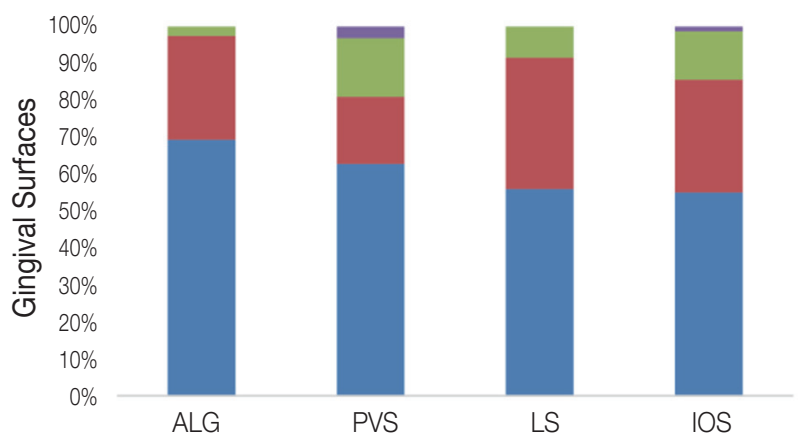

-Accurate $\quad$ Minor Deviation $\quad=$ Moderate Deviation $\quad$ Major Deviation
B

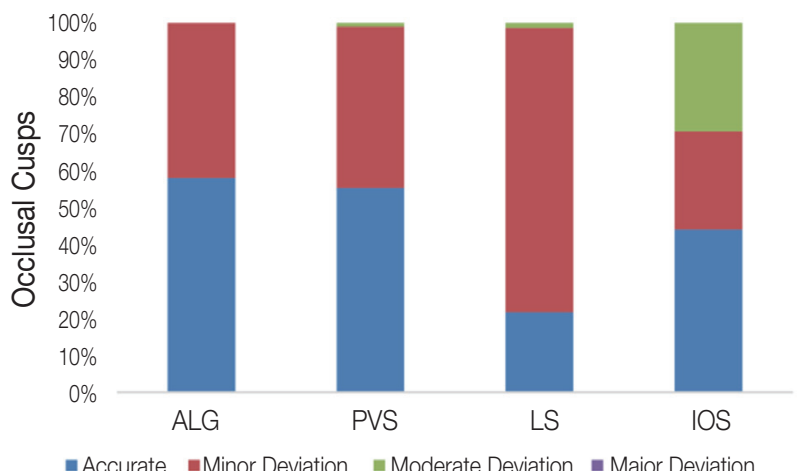

D

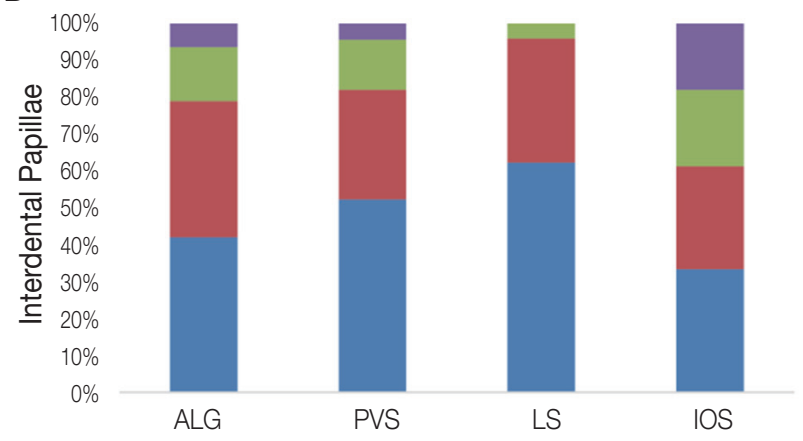

=Accurate $\quad$ Minor Deviation $=$ Moderate Deviation $=$ Major Deviation

$\mathbf{F}$

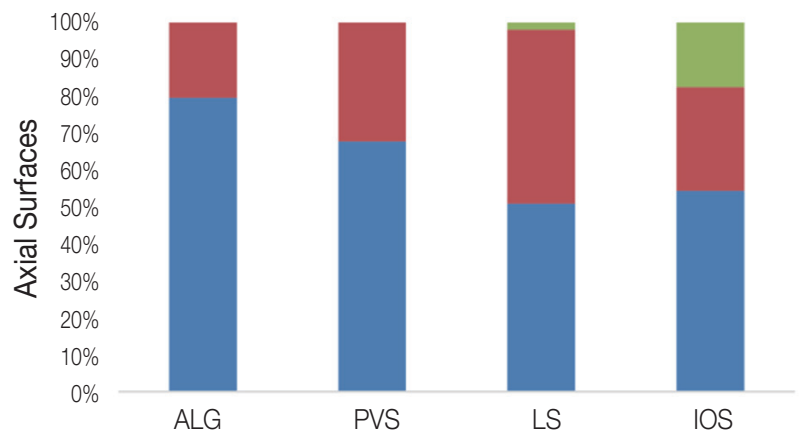

=Accurate $\quad$ Minor Deviation $\quad$ moderate Deviation $=$ Major Deviation
Fig. 4. The frequency of deviation of the different surfaces among the 4 groups: (A) Occlusal fossae, (B) Occlusal cusps, (C) Incisal edges, (D) Interdental papillae, (E) Proximal surfaces, (F) Axial surfaces, (G) Gingival surface. 
alence of more severe distortion. There were statistical differences among all of them, except between the PVS and LS $(P=.05)$. In relation to the cusp tips (Fig. 4B), there was a significant difference among the groups, except between ALG and PVS $(P=.19)$ and both of them were superior to IOS and LS. The LS had the greatest amount of deviation, while the IOS was distinguished by a more severe deviation. A similar pattern was observed for the incisal edges (Fig. 4C). There were differences among the groups, except between ALG and PVS $(P=.53)$, ALG and IOS $(P=.41)$, and PVS and IOS $(P=.84)$. The LS was clearly more inferior compared to the other groups.

For the interdental papillae (Fig. 4D), a significant difference was observed among all the groups, except between PVS and ALG $(P=.70)$. The best outcome was shown for LS while the worst was shown for IOS. In relation to the proximal surfaces (Fig. 4E), axial surfaces (Fig. 4F), and gingival surfaces (Fig. 4G), the groups were significantly different. ALG tended to have the superior outcome followed by PVS, LS, and IOS.

The trueness values of all the teeth for ALG, PVS, LS, and IOS were $65.6 \pm 21.6 \mu \mathrm{m}, 57.1 \pm 15.6 \mu \mathrm{m}, 38.1 \pm 4.4$ $\mu \mathrm{m}$, and $37.9 \pm 4.1 \mu \mathrm{m}$ respectively (Fig. 5). The LS and IOS were the most accurate followed by PVS and ALG. The differences in accuracy were significant $(P<.001)$, except between the IOS and the LS $(P=.33)$. In addition, it is clear that the LS and IOS have much less variation than PVS and ALG, while the ALG had the greatest variation. Likewise, the superior trueness of LA and IOS was observed for each tooth category.

There was a significant interaction between the impression method and the tooth category $(P<.001)$. Table 1 summarizes the outcome of the segmented teeth for each group. There was a tendency for better trueness of the more posterior regions than the anterior regions for ALG and, to a lesser extent, PVS (Fig. 6). The greatest variation was observed for ALG. Significant differences for the ALG group were observed among all the teeth, except between premolars and molars. For the PVS group, the differences among the teeth were significant except between canines and incisors. While the teeth for the LS and IOS had trueness within a similar range, statistical differences were observed. For the LS group, significant differences existed among all the teeth except between incisors and premolars, and between canines and premolars. The significant differences in the IOS group occurred between canines and premolars and between premolars and molars.

\section{DISCUSSION}

This study indicated that the different techniques of producing casts have different accuracy for the whole arch and the different spans of teeth. Thus, the hypothesis that all the workflows produce casts of similar accuracy is rejected. For the whole arch accuracy, the IOS workflow had the most inferior trueness and precision. On the contrary, for a single tooth, the two digital workflows were superior compared with the conventional workflows. The difference in the outcome is likely to be due to differences in the sources of errors such as the manufacturing approach and materials manipulation. Trueness and precision are frequently used variables to virtually evaluate the errors between digital models. However, due to their numerical variables and averaging surface errors, they do not provide information on the pattern of deviation and the severity of the errors. Therefore, qualitative evaluation was implemented and modified in this study to measure the frequency and severity of the errors.

The superiority of the conventional PVS impressions can be attributed to the favorable material properties such as the accuracy, surface details, dimensional stability, tear resistance, and elastic recovery in comparison to any other impression material. ${ }^{2-5}$ In addition, the use of custom tray reduced the bulk of the PVS material which is prone to irreversible material distortion. ${ }^{2-5}$ Pouring the PVS impres-

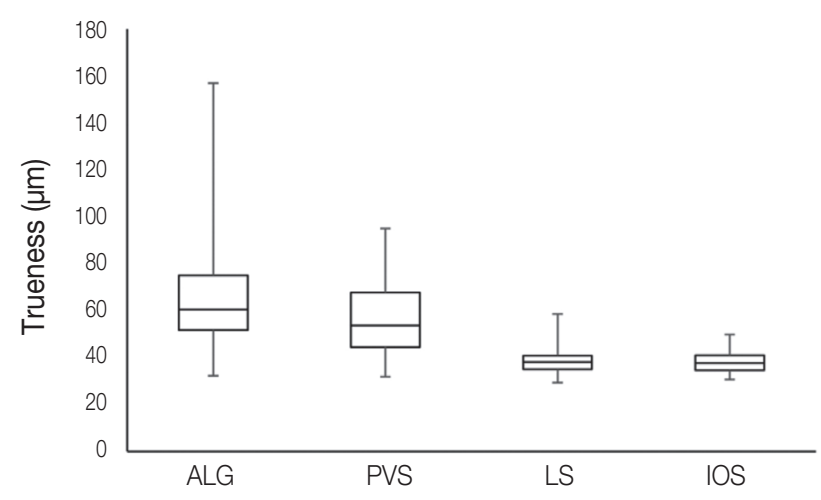

Fig. 5. Box plot diagram illustrating the teeth trueness of the different groups.

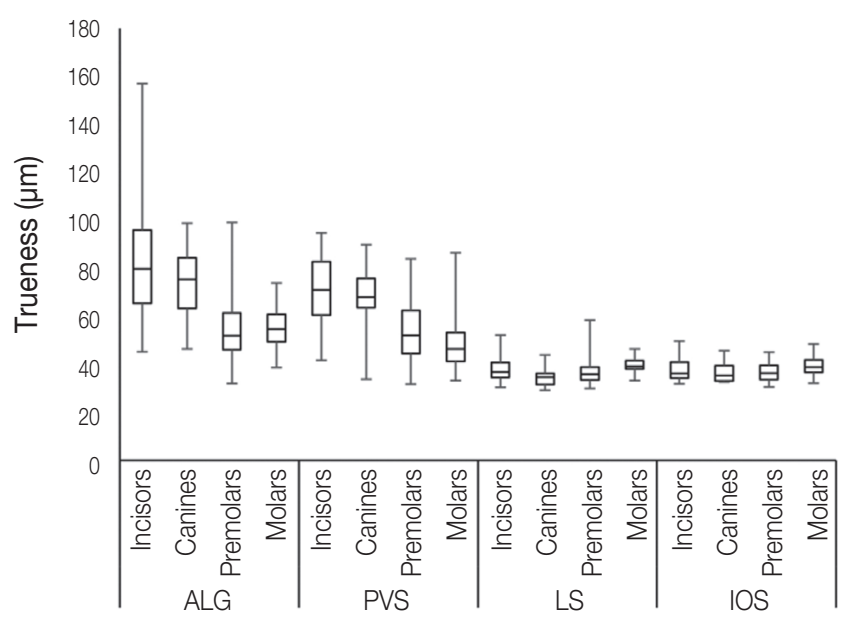

Fig. 6. Box plot diagram illustrating the trueness of different teeth categories of all the groups. 
Table 1. Summary of means and standard deviations (SD) of the segmented teeth for each group (Unit: $\mu \mathrm{m})$

\begin{tabular}{|c|c|c|c|c|c|c|c|c|}
\hline & \multicolumn{8}{|c|}{ Teeth } \\
\hline & \multicolumn{2}{|c|}{ Incisors } & \multicolumn{2}{|c|}{ Canines } & \multicolumn{2}{|c|}{ Premolars } & \multicolumn{2}{|c|}{ Molars } \\
\hline & Mean & SD & Mean & SD & Mean & SD & Mean & SD \\
\hline ALG & 85.6 & 28.0 & 73.8 & 13.1 & 56.3 & 15.7 & 55.6 & 8.1 \\
\hline$P$ values & \multicolumn{3}{|c|}{$\begin{array}{c}\text { Incisors vs canines }=.04 \\
\text { Canines vs premolars }=.000\end{array}$} & \multicolumn{2}{|c|}{$\begin{array}{c}\text { Incisors vs premolars }=.000 \\
\text { Canines vs molars }=.000\end{array}$} & \multicolumn{3}{|c|}{$\begin{array}{l}\text { Incisors vs molars }=.000 \\
\text { Premolars vs molars }=.38\end{array}$} \\
\hline PVS & 70.2 & 14.7 & 67.2 & 14.6 & 52.9 & 12.1 & 47.7 & 9.9 \\
\hline$P$ values & \multicolumn{3}{|c|}{$\begin{array}{c}\text { Incisors vs canines }=.23 \\
\text { Canines vs premolars }=.000\end{array}$} & \multicolumn{2}{|c|}{$\begin{array}{l}\text { Incisors vs premolars }=.000 \\
\text { Canines vs molars }=.000\end{array}$} & \multicolumn{3}{|c|}{$\begin{array}{l}\text { Incisors vs molars }=.000 \\
\text { Premolars vs molars }=.01\end{array}$} \\
\hline LS & 38.1 & 4.8 & 34.7 & 3.8 & 36.9 & 4.9 & 39.9 & 2.8 \\
\hline$P$ values & \multicolumn{3}{|c|}{$\begin{array}{l}\text { Incisors vs canines }=.01 \\
\text { Canines vs premolars }=.08\end{array}$} & \multicolumn{2}{|c|}{$\begin{array}{l}\text { Incisors vs premolars }=.14 \\
\text { Canines vs molars }=.000\end{array}$} & \multicolumn{3}{|c|}{$\begin{array}{c}\text { Incisors vs molars }=.02 \\
\text { Premolars vs molars }=.000\end{array}$} \\
\hline IOS & 37.7 & 4.6 & 36.7 & 3.9 & 36.6 & 3.6 & 39.2 & 3.8 \\
\hline \multirow[t]{2}{*}{$P$ values } & \multicolumn{3}{|c|}{$\begin{array}{c}\text { Incisors vs canines }=.19 \\
\text { Canines vs premolars }=.46\end{array}$} & \multicolumn{2}{|c|}{$\begin{array}{l}\text { Incisors vs premolars }=.10 \\
\text { Canines vs molars }=.01\end{array}$} & \multicolumn{3}{|c|}{$\begin{array}{l}\text { Incisors vs molars }=.08 \\
\text { Premolars vs molars }=.00\end{array}$} \\
\hline & & & & nes & Prem & & & \\
\hline $\begin{array}{l}P \text { values after } \\
\text { comparing each } \\
\text { technique within } \\
\text { every tooth } \\
\text { category }\end{array}$ & $\begin{array}{l}\text { ALG } \\
\text { ALG } \\
\text { ALG } \\
\text { PVS } \\
\text { PVS } \\
\text { LS V }\end{array}$ & $\begin{array}{l}.001 \\
000 \\
.000 \\
000 \\
.000 \\
6\end{array}$ & $\begin{array}{l}A L G \text { V } \\
A L G \text { V } \\
A L G \text { V } \\
P V S \text { V } \\
P V S \text { V } \\
L S \text { V }\end{array}$ & $\begin{array}{l}S=.09 \\
S=.000 \\
S=.000 \\
=.000 \\
S=.000 \\
=.06\end{array}$ & $\begin{array}{l}\text { ALG vs P } \\
\text { ALG vs } L \\
\text { ALG vs IC } \\
\text { PVS vs LS } \\
\text { PVS vs IC } \\
\text { LS vs IOS }\end{array}$ & $\begin{array}{l}.13 \\
000 \\
.000 \\
000 \\
.000 \\
33\end{array}$ & $\begin{array}{l}\text { ALG V } \\
A L G V \\
A L G V \\
P V S V \\
P V S V \\
L S \vee S\end{array}$ & $\begin{array}{l}.000 \\
000 \\
.000 \\
000 \\
.000 \\
9\end{array}$ \\
\hline
\end{tabular}

sion with the minimal expansion type IV dental stone will further reduce the cast distortion. ${ }^{13}$ In accordance with the present study, several studies consistently reported a superior accuracy of whole arch PVS impressions over IOS ${ }^{6-10}$ and $3 \mathrm{D}$ printed casts. ${ }^{12-14}$ Nevertheless, in the present study, the observed whole arch accuracy of PVS was not significantly greater than the ALG group, which is contrary to earlier laboratory and clinical studies. ${ }^{8,10}$ The similarity between the 2 conventional impression techniques can be partly related to the laboratory experiment set-up and the immediate pouring of ALG impressions, which may reduce the distortion of the ALG impressions. More importantly, this can be attributed to the dentate nature of the master model that has intact teeth with natural undercuts, which subjects the PVS material to strains as the impression is separated from the arch. This may lead to more distortion for the PVS impressions of this study as compared to the impressions of prepared teeth with no undercuts. ${ }^{5}$ The clinical similarity of whole arch impressions for ALG and PVS was reported by a clinical study by Fokkinga et al., ${ }^{1}$ which evaluated the clinical fit of RPD fabricated after ALG or PVS impressions. No significant differences were found in the clinical fit of metal frameworks fabricated from casts produced from ALG and PVS. However, the superiority of PVS became apparent for single teeth accuracy, which confirms its superior surface details and its suitability for making accurate prostheses to fit the preparations.

The qualitative evaluation revealed that the conventional impressions were affected by localized distortion predominantly on the occlusal fossa and interdental papilla regions, which has been observed by earlier studies. ${ }^{8,10,13}$ The sensitivity of the occlusal fossa regions may be related to the wetting of the occlusal surfaces and filling the anatomical details of the posterior teeth. In addition, conventional impressions showed greater errors at the interdental papilla regions and the gingival surface, which can be attributed to the permanent deformation of impression material as it is removed from the undercut regions..$^{2-5}$ This was further confirmed with the enlarged dimension at the error sites of the final casts after conventional impressions. On the other hand, the conventional impressions were generally accurate on the occlusal cusps and incisal edges, and proximal surfaces and axial surfaces, which do not interfere with material flow and are less affected by the removal of the impression. In addition, the material sensitivity of arch and teeth morphology explains why inferior accuracy was observed for incisors followed by canines and posterior teeth. As the anterior teeth are longer and tend to have higher and deeper undercuts than the posterior teeth, they challenge the impression material and prolong the duration of material distortion as the impression is removed from the arch. ${ }^{5}$

The LS casts of the present study were narrower than 
the original model. This error appeared to be related to shrinkage associated with the additive manufacturing process and the inevitable minimal shrinkage of each layer during polymerization, and the accumulated errors of layering the sequential layers. The shrinkage was observed for different types of 3D printers by numerous studies. ${ }^{12,15,16}$ As the layers are contracted during curing, this induces stresses within the produced cast, which leads to dimensional distortion. ${ }^{13}$ The effect of shrinkage was more obvious for the posterior region than for the anterior region. This can be attributed to the smoother surface of the anterior teeth and their location within the middle of the arch. ${ }^{14}$ Contrary to the conventional workflows, this distortion pattern had led to minimal but generalized deviation from the virtual master model affecting primarily the proximal, axial surfaces, occlusal cusps, and incisal edges. In addition, the vertical layering will result in a staircase effect that will mainly affect inclined and corrugated regions, which can further contribute to loss of definition. ${ }^{11}$ The concern that was raised about $3 \mathrm{D}$ printed casts is that it may be acceptable for overall dimensions, but may miss some critical areas and fine details necessary for the quality of the prosthesis. ${ }^{18}$ For example, Anadioti et al. reported inferior marginal accuracy for pressed ceramic crowns on printed casts. ${ }^{19}$ However, the clinical implication of the staircase effect on the quality of the prosthesis is yet to be determined.

The inferior arch accuracy and the distortion of the IOS casts can be primarily attributed to the IOS step, which further accentuated the errors from 3D printing. The IOS scanning inaccuracy can be related to a smaller scanning field and missing shadowed surfaces. Consequently, multiple imaging and stitching are mandatory to generate a continuous virtual arch. Each stitching procedure is associated with an error that may become more pronounced at the most distant site. ${ }^{7,810,17}$ This was observed from the qualitative evaluation of this study, where the posterior regions seemed to accumulate more generalized errors and surface mismatch from the original virtual model. In accordance with this study, this observation was consistently reported by earlier papers on IOS virtual image accuracy ${ }^{9,21-23}$ and studies on $3 \mathrm{D}$ printed casts from IOS images. ${ }^{13,14}$ On the contrary, laboratory scanning takes multiple large field images of the whole arch and combines them in a single image in an automated process. This means that the overall dimensions of the whole arch is registered by each laboratory scan imaging, which may explain the superior accuracy observed for the LS casts than IOS casts in this study and previous studies. ${ }^{9,10,12,15,23,24}$

On the other hand, IOS single tooth accuracy was superior to conventional methods and similar to LS. This can be attributed to the reduced effect of image stitching and the IOS camera accuracy that is comparable to laboratory scanner accuracy. Other studies found similar outcomes in which the accuracy of short span scanning was comparable to conventional impressions ${ }^{25,26}$ and laboratory scanning., ${ }^{7,13,24}$ A comparable finding was reported by $\mathrm{Ng}$ et al. ${ }^{27}$ who found better fit for crowns fabricated by full digital workflow using IOS than PVS impressions. Therefore, while the IOS yields inferior accuracy for whole arch scanning, it is more accurate for single tooth or short span scanning. Thus, it can be speculated that IOS is reliable for short span scanning. For whole arch scanning, earlier reports suggested that IOS is suitable for diagnostic purposes, such as diagnostic wax-up and orthodontic planning. ${ }^{17,28}$ However, it may not exhibit sufficient accuracy for definitive whole arch prosthesis fabrication. Thus, at this stage, the complete digital workflow, despite its clear advantages, cannot reliably replace the conventional workflow for large prostheses fabrication. This is important as casts are needed for large prostheses fabrication, and customizing the occlusion, contour, and proximal areas. Inferior accuracy may translate clinically into extensive chairside adjustment.

Nevertheless, this study is limited in its laboratory nature that do not simulate the true nature of oral environment such as intraoral access, saliva, reflection, and mobility of soft tissue, which pose more challenges to IOS and conventional impression techniques. The application of powder prior to scanning is a recommendation by several IOS system manufacturers to reduce the light reflection from metallic restorations. However, the beneficial effect of powder application is questioned by recent literature, ${ }^{8,11}$ and the clinicians should be aware of the necessity of maintaining thin powder layer during scanning of the whole arch. Inconsistent or thick powder layer may potentially introduce errors to the scanning. ${ }^{11}$ Therefore, clinical studies are always desirable to confirm the outcome of the present study.

\section{CONCLUSION}

Within the limitations of the present study, it can be concluded that for whole arch accuracy (precision and trueness), the IOS was the most inferior, while the other 3 techniques were similar. The digital workflows were associated with errors of the casts at the posterior region, which was worse for the IOS workflow. The conventional impression distortion pattern was related to the material properties. Digital workflows were clearly more accurate for single teeth reproduction than conventional impressions.

\section{ORCID}

Jaafar Abduo https://orcid.org/0000-0003-3392-8641

\section{ACKNOWLEDGMENTS}

The author acknowledges the work of Drs. Dorothy Wang, Joanna Wang, Lisa Wang, William Wang, Xue Ying Wang, Zibo Wangding in conducting the pilot aspect of the study.

The author would like to acknowledge the technical support Mr. Clay Taylor and Mr. Attila Gergely.

The $3 \mathrm{D}$ printing was completed by XYZ Dental, Melbourne. 


\section{REFERENCES}

1. Fokkinga WA, Witter DJ, Bronkhorst EM, Creugers NH. Clinical fit of partial removable dental prostheses based on alginate or polyvinyl siloxane impressions. Int J Prosthodont 2017;30:33-7.

2. Gordon GE, Johnson GH, Drennon DG. The effect of tray selection on the accuracy of elastomeric impression materials. J Prosthet Dent 1990;63:12-5.

3. Rueda LJ, Sy-Muñoz JT, Naylor WP, Goodacre CJ, Swartz ML. The effect of using custom or stock trays on the accuracy of gypsum casts. Int J Prosthodont 1996;9:367-73.

4. Ceyhan JA, Johnson GH, Lepe X. The effect of tray selection, viscosity of impression material, and sequence of pour on the accuracy of dies made from dual-arch impressions. J Prosthet Dent 2003;90:143-9.

5. de Araujo PA, Jorgensen KD. Effect of material bulk and undercuts on the accuracy of impression materials. J Prosthet Dent 1985;54:791-4.

6. Kuhr F, Schmidt A, Rehmann P, Wöstmann B. A new method for assessing the accuracy of full arch impressions in patients. J Dent 2016;55:68-74.

7. Atieh MA, Ritter AV, Ko CC, Duqum I. Accuracy evaluation of intraoral optical impressions: A clinical study using a reference appliance. J Prosthet Dent 2017;118:400-5.

8. Ender A, Attin T, Mehl A. In vivo precision of conventional and digital methods of obtaining complete-arch dental impressions. J Prosthet Dent 2016;115:313-20.

9. Flügge TV, Schlager S, Nelson K, Nahles S, Metzger MC. Precision of intraoral digital dental impressions with iTero and extraoral digitization with the iTero and a model scanner. Am J Orthod Dentofacial Orthop 2013;144:471-8.

10. Ender A, Mehl A. In-vitro evaluation of the accuracy of conventional and digital methods of obtaining full-arch dental impressions. Quintessence Int 2015;46:9-17.

11. Abduo J, Lyons K, Bennamoun M. Trends in computer-aided manufacturing in prosthodontics: a review of the available streams. Int J Dent 2014;2014:783948.

12. Al-Imam H, Gram M, Benetti AR, Gotfredsen K. Accuracy of stereolithography additive casts used in a digital workflow. J Prosthet Dent 2018;119:580-5.

13. Sim JY, Jang Y, Kim WC, Kim HY, Lee DH, Kim JH. Comparing the accuracy (trueness and precision) of models of fixed dental prostheses fabricated by digital and conventional workflows. J Prosthodont Res 2019;63:25-30.

14. Jin SJ, Kim DY, Kim JH, Kim WC. Accuracy of dental replica models using photopolymer materials in additive manufacturing: In vitro three-dimensional evaluation. J Prosthodont 2019;28:e557-62.

15. Wesemann C, Muallah J, Mah J, Bumann A. Accuracy and efficiency of full-arch digitalization and 3D printing: A comparison between desktop model scanners, an intraoral scanner, a CBCT model scan, and stereolithographic 3D printing. Quintessence Int 2017;48:41-50.

16. Ishida Y, Miyasaka T. Dimensional accuracy of dental casting patterns created by 3D printers. Dent Mater J 2016;35:250-6.

17. Abduo J, Elseyoufi M. Accuracy of intraoral scanners: A sys- tematic review of influencing factors. Eur J Prosthodont Restor Dent 2018;26:101-21.

18. Eftekhar Ashtiani R, Nasiri Khanlar L, Mahshid M, Moshaverinia A. Comparison of dimensional accuracy of conventionally and digitally manufactured intracoronal restorations. J Prosthet Dent 2018;119:233-8.

19. Anadioti E, Aquilino SA, Gratton DG, Holloway JA, Denry I, Thomas GW, Qian F. 3D and 2D marginal fit of pressed and CAD/CAM lithium disilicate crowns made from digital and conventional impressions. J Prosthodont 2014;23:610-7.

20. ISO 5725-1. Accuracy (trueness and precision) of measurement methods and results - Part 1: General principles and definitions. International Standards Organization (ISO); Geneva; Switzerland, 1994. Available at:https://www.iso.org/ standard/11833.html. Accessed August, 2018.

21. Ender A, Mehl A. Accuracy of complete-arch dental impressions: a new method of measuring trueness and precision. J Prosthet Dent 2013;109:121-8.

22. Jeong ID, Lee JJ, Jeon JH, Kim JH, Kim HY, Kim WC. Accuracy of complete-arch model using an intraoral video scanner: An in vitro study. J Prosthet Dent 2016;115:755-9.

23. Atieh MA, Ritter AV, Ko CC, Duqum I. Accuracy evaluation of intraoral optical impressions: A clinical study using a reference appliance. J Prosthet Dent 2017;118:400-5.

24. Su TS, Sun J. Comparison of repeatability between intraoral digital scanner and extraoral digital scanner: An in-vitro study. J Prosthodont Res 2015;59:236-42.

25. Lee JJ, Jeong ID, Park JY, Jeon JH, Kim JH, Kim WC. Accuracy of single-abutment digital cast obtained using intraoral and cast scanners. J Prosthet Dent 2017;117:253-9.

26. Cho SH, Schaefer O, Thompson GA, Guentsch A. Comparison of accuracy and reproducibility of casts made by digital and conventional methods. J Prosthet Dent 2015;113: 310-5.

27. $\mathrm{Ng}$ J, Ruse D, Wyatt C. A comparison of the marginal fit of crowns fabricated with digital and conventional methods. J Prosthet Dent 2014;112:555-60.

28. Rossini G, Parrini S, Castroflorio T, Deregibus A, Debernardi CL. Diagnostic accuracy and measurement sensitivity of digital models for orthodontic purposes: A systematic review. Am J Orthod Dentofacial Orthop 2016;149:161-70. 